Revue d'histoire de l'Amérique française

Q6 REVUE D.HISTOIRE DE L'AMÉRIQUE FRANÇAISE

\title{
Visages de la paternité au Québec, 1900-1960
}

Peter Gossage

Volume 70, numéro 1-2, été-automne 2016

URI : https://id.erudit.org/iderudit/1038289ar

DOI : https://doi.org/10.7202/1038289ar

Aller au sommaire du numéro

\section{Éditeur(s)}

Institut d'histoire de l'Amérique française

\section{ISSN}

0035-2357 (imprimé)

1492-1383 (numérique)

Découvrir la revue

\section{Citer cet article}

Gossage, P. (2016). Visages de la paternité au Québec, 1900-1960. Revue d'histoire de l'Amérique française, 70(1-2), 53-82.

https://doi.org/10.7202/1038289ar

\section{Résumé de l'article}

L'auteur poursuit une réflexion historique sur les pères et la paternité au Québec entre 1900 et 1960, inspirée par l'historiographie internationale dans ce domaine et alimentée par quatre corpus des sources distincts et complémentaires. S'inscrivant dans le cadre de cette réflexion et de cette recherche plus larges, son propos ici vise dans un premier temps à offrir une esquisse rapide d'un schéma en trois " modèles » successifs - le père pourvoyeur, le père éducateur et le nouveau père - qui structurent, selon sa lecture, l'historiographie des pères au Québec depuis le XIX ${ }^{\mathrm{e}}$ siècle. Par la suite, il propose le concept des « visages de la paternité » comme nouvelle grille d'analyse, empruntée aux historiens John Demos et Robert Rutherdale, qui favorise une interprétation plus riche, plus nuancée et moins linéaire de cette histoire. Au final, l'auteur puise dans deux corpus de sources, à savoir les récits de vie et les annonces publicitaires, pour démontrer à partir de quatre exemples (le père spirituel, le père disciplinaire, le père prévoyant et le père sportif) à quel point ce concept des visages permet de faire avancer la discussion. C'est dire en même temps qu'il est nécessaire et très possible de situer les pères québécois par rapport à une gamme de rôles, d'attentes et de responsabilités dynamique dans le temps, variable dans l'espace social ou géographique et beaucoup plus vaste et complexe que généralement reconnue. 


\title{
Visages de la paternité au Québec, I900-I960'
}

\author{
Peter Gossage \\ Université Concordia
}

RÉSUMÉ • L'auteur poursuit une réflexion historique sur les pères et la paternité au Québec entre 1900 et 1960, inspirée par l'historiographie internationale dans ce domaine et alimentée par quatre corpus des sources distincts et complémentaires. S'inscrivant dans le cadre de cette réflexion et de cette recherche plus larges, son propos ici vise dans un premier temps à offrir une esquisse rapide d'un schéma en trois «modèles» successifs - le père pourvoyeur, le père éducateur et le nouveau père - qui structurent, selon sa lecture, l'historiographie des pères au Québec depuis le XIXe siècle. Par la suite, il propose le concept des «visages de la paternité» comme nouvelle grille d'analyse, empruntée aux historiens John Demos et Robert Rutherdale, qui favorise une interprétation plus riche, plus nuancée et moins linéaire de cette histoire. Au final, l'auteur puise dans deux corpus de sources, à savoir les récits de vie et les annonces publicitaires, pour démontrer à partir de quatre exemples (le père spirituel, le père disciplinaire, le père prévoyant et le père sportif) à quel

1. Ce texte résume certains résultats d'un projet plus large ayant bénéficié d'une subvention du CRSH (Pères et paternité au Québeec, 1900-1960) et d'une contribution d'appoint de l'Université Concordia, fournie dans le cadre de son entente de partenariat avec le Centre interuniversitaire d'études québécoises (CIÉQ). Je tiens à remercier Louise Bienvenue et l'équipe de rédaction de la revue ainsi que les deux évaluateurs anonymes pour leurs encouragements et leurs conseils judicieux. J'exprime par ailleurs ma reconnaissance envers un groupe remarquable d'étudiantes et d'étudiants ayant participé à ce projet depuis ses débuts à titre d'auxiliaires de recherche, dont Marie-Laurence B.-Beaumier, Magaly Brodeur, Jason Butters, Antoine Guillemette, Abby Mahon, Lisa Moore, Thierry Robert, Michelle Roy et Guillaume Saudrais. Des éléments de ce texte ont été présentés à l’Université Laval, à l'IHAF, et à deux reprises devant des groupes d'étudiants de maîtrise à l'Université de Sherbrooke. Ces échanges m'ont permis de clarifier mon propos et de pousser plus loin ma réflexion sur la paternité québécoise et je reconnais chaleureusement ma dette envers ceux et celles qui ont participé. Merci enfin à Robert Rutherdale, ami et collaborateur à qui je dois la métaphore des visages, et surtout à Annmarie Adams, partenaire dans la vie et dans la parentalité, à qui je dois tout le reste. 
point ce concept des visages permet de faire avancer la discussion. C'est dire en même temps qu'il est nécessaire et très possible de situer les pères québécois par rapport à une gamme de rôles, d'attentes et de responsabilités dynamique dans le temps, variable dans l'espace social ou géographique et beaucoup plus vaste et complexe que généralement reconnue.

ABStract - The author draws here on his exploration into the history of fathers and fatherhood in Quebec between 1900 and 1960, a project inspired by the international literature in the field and sustained by four distinct and complementary sets of primary sources. Framed within this broader initiative, the article begins with a sketch of a standard sequence of three successive « models »- the breadwinner, the teacher, and the new father - that structures, in his reading, most of the literature on Quebec fathers since the $19^{\text {th }}$ century. With that in place, he proposes the 'faces of fatherhood' as a new conceptual framework, borrowed from historians John Demos and Robert Rutherdale, that can foster a richer, more nuanced, and less linear interpretation of the paternal experience over time. In the end, drawing on two sets of primary sources (life writing and commercial advertising), the author uses the examples of four specific «faces 》 - the spiritual father; the disciplinarian father; the farsighted father; and the father as sportsman - to demonstrate the power of this concept to move the discussion forward. It is quite possible and indeed necessary, in other words, to situate Quebec fathers with respect to a much wider and more complex range of roles, expectations, and responsibilities than is generally allowed in the literature, incorporating detailed attention to change over time and to variations by geographic and social location.

L 'étude du masculin n'est plus, en 2016, le parent pauvre de l'histoire des identités sexuées au Québec². Nous avons maintenant de bonnes études sur une foule de sujets dans ce domaine, que ce soit les rites de passage vers le statut d'homme et de voyageur dans la traite des fourrures; les collèges classiques d'autrefois, vus comme «l'une des pépinières canoniques du masculin au Québec»; la violence politique, ce lieu d'expression des valeurs viriles au XIX ${ }^{\mathrm{e}}$ siècle; le rapport entre les identités masculines et le discours nationaliste à l'époque de Bourassa et de Groulx; la puissance paternelle comme concept juridique fondamental, mais en perte de vitesse au $\mathrm{XX}^{\mathrm{e}}$ siècle; voire les rapports complexes entre paternité et citoyenneté dans les années 1940 et $1950^{3}$. Et il y a bien d'autres travaux

2. Jeffery Vacante, dans un article publié en 2006, déplorait la lenteur du développement d'un intérêt pour l'étude du masculin chez les historiens du Québec: «Liberal Nationalism and the Challenge of Masculinity Studies in Quebec», Left History, 11, 2 (2006): 96-117.

3. Carolyn Podruchny, Les Voyageurs et leur monde: Voyageurs et traiteurs de fourrures en Amérique du Nord, trad. Anne-Hélène Kerbiriou (Québec, Les Presses de l’Université Laval, 2009); Louise Bienvenue, Ollivier Hubert et Christine Hudon, Le collège classique pour garçons. Études historiques sur une institution québécoise disparue (Montréal, Fides, 2014), surtout la partie 3, "In amore fraternitatis: la construction du 
en cours qui avancent sur la voie tracée par Joan Scott et les autres historiennes féministes qui nous encouragent à étudier le genre - soit les rapports sociaux de sexe-comme une catégorie fondamentale de l'expérience historique, y compris celle des hommes ${ }^{4}$.

L'étude rétrospective des hommes dans leur milieu domestique, et plus précisément dans leur rôle de père - l'ensemble des enjeux, autrement dit, qui entourent la parentalité masculine - n'est pas non plus complètement en friche au Québec ${ }^{5}$. Ce sont les littéraires qui en ont posé les premiers jalons, faisant ressortir l'importance de la figure paternelle dans la littérature canadienne-française puis québécoise : personnage souvent "faible, irresponsable, mécontent ou absent " pour reprendre une formulation de Patricia Smart ${ }^{6}$. Du côté des sciences sociales, l'intérêt se développe au rythme des mutations majeures vécues récemment au sein des familles contemporaines. Une large part de cette production est inspirée de soucis socio-sanitaires, visant à promouvoir des interventions éclairées et efficaces auprès des individus à risque et des familles en difficulté ${ }^{7}$. Mais

masculin", 235-388 (citation tirée de l'introduction, 13); Dan Horner, " "Shame upon you as men!": Contesting Authority in the Aftermath of Montreal's Gavazzi Riot ", Histoire sociale/Social History 44, 87 (mai 2011): 29-52; Jeffery Vacante, The Search for Manhood: Locating Masculine Identity in Early TwentiethCentury Quebec, Thèse de doctorat (histoire), University of Western Ontario, 2005 ; J. Vacante, «Evolving Racial Identity and the Consolidation of Men's Authority in Early Twentieth-Century Quebec», Canadian Historical Review, 88, 3 (septembre 2007): 413-438; Magda Fahrni, Household Politics: Montreal Families and Postwar Reconstruction (Toronto, University of Toronto Press, 2005) et notamment le chapitre 6, «In the Streets: Fatherhood and Public Protest». Les études portant sur le statut juridique du père sont citées plus loin à la note 9 (textes de Cliche, Fish et Saudrais) tout comme celle de Duhaime, portant sur les modèles paternels durant l'après-guerre immédiat.

4. La référence à l'article phare de Scott, dans sa version française, est la suivante: Joan Scott, «Genre: une catégorie utile d'analyse historique». Cahiers du GRIF, 37-38 (printemps 1988): 41-67. De nombreuses études actuellement en cours partent d'une telle conceptualisation (relationnelle) des rapports de genre pour étudier des aspects particuliers du masculin dans le contexte québécois. À titre d'exemple, mentionnons deux thèses de doctorat en préparation, celle de Sonya Roy à l'Université McGill ("You say that you are a single man. You must realize that work is first given to the married men": Les hommes célibataires montréalais et la crise économique des années 1930) et celle de Marie-Laurence B. Beaumier à l'Université Laval: Une incursion au cour du cycle de vie familial: pratiques et expériences parentales en milieu populaire québécois, 1945-1977.

5. Nous empruntons l'expression "parentalité masculine» à M.-L. B. Beaumier, op. cit.. Voir aussi son article, "Parentalité, genre et âges de la vie: croisements et perspectives de recherche ", à paraître dans la revue Enfance, Familles, Générations.

6. Patricia Smart, De Marie de l'Incarnation à Nelly Arcand. Se dire, se faire par l'écriture féminine (Montréal, Boréal, 2014): 313. Voir aussi Lori Saint-Martin, Au-delà du nom: La question du père dans la littérature québécoise actuelle (Montréal, Presses de l’Université de Montréal, 2010).

7. L'une des contributions les plus marquantes est celle du psychanalyste Guy Corneau, qui postulait en 1989 l'existence d'une «crise de la masculinité», conséquence des mutations socio-sexuelles de l'époque, dont une fragilisation des modèles masculins et du rapport entre père et fils. Guy Corneau, Père manquant, fils manqué (Montréal, Éditions de l’Homme, 1989). Pour un survol récent sous forme d'ouvrage collectif, voir Diane Dubeau, Annie Devault et Gilles Forge (dir.), La paternité au XXI siècle (Québec, Les Presses de l’Université Laval, 2009). 
certains chercheurs se penchent aussi sur l'évolution des modèles paternels à travers le temps, généralement avec un accent sur la période récente et sans recours systématique aux sources primaires ${ }^{8}$.

Quant aux spécialistes de l'histoire sociale, ou parfois de la sociologie historique, ils sont plus nombreux, depuis les années 1990, à se pencher sur les pères québécois du passé. Ils le font, d'abord, dans le cadre d'études ciblées, préparées sous forme de mémoire ou de thèse, dont certains ont fait l'objet d'importants articles scientifiques ${ }^{9}$. Ils le font ensuite par la bande, dans des études portant sur des sujets plus larges; nous pouvons apprendre beaucoup sur les pères québécois, par exemple, en lisant des travaux récents sur la mémoire collective d'un village saguenéen, sur les attitudes envers l'enfance au début du $\mathrm{XX}^{\mathrm{e}}$ siècle, sur la zone grise et mouvante entre les punitions corporelles et la violence domestique, voire sur la doctrine sociale catholique dans les décennies qui préparent la Révolution tranquille ${ }^{10}$.

Pour notre part, nous poursuivons depuis un moment une réflexion sur les pères et la paternité au Québec entre 1900 et 1960, inspirée par l'historiographie internationale dans ce domaine et alimentée par quatre corpus

8. Voir Jean-Martin Deslauriers, Judith Gaudet et Dominic Bizot, «Le rôle du père, de la NouvelleFrance au Québec de demain», dans ibid., 15-38; Josée Saint-Denis et Nérée Saint-Amand, "Les pères dans l'histoire: un rôle en évolution", Reflets : revue d'intervention sociale et communautaire 16, 1 (2010): 32-61.

9. Cynthia S. Fish, Images and Reality of Fatherhood: A Case Study of Montreal's Protestant Middle Class, 1870-1914, thèse de doctorat (histoire), Université McGill, 1991; Cynthia Fish, «La puissance paternelle et les cas de garde d'enfants au Québec, 1866-1928 ", Revue d'histoire de l'Amérique française 57, 4 (printemps 2004): 509-533 ; Elizabeth Wright, La paternité au Québec de 1890 à 1965 : une lecture d'ouvrages sociologiques, mémoire de maîtrise (sociologie), Université Laval, 1997; Ginette Girard, Les pratiques de travail dans la société patrimoniale québécoise du dix-neuvième siècle: ascendant de la mère et exclusion du père?, mémoire de maîtrise (sociologie), Université Laval, 1997; Vincent Duhaime, La construction du père québécois : le discours du mouvement familial et l'expérience des pères, 1945-1965, mémoire de maîtrise (histoire), Université de Montréal, 2000; Vincent Duhaime, "Les pères ont ici leur devoir” : Le discours du mouvement familial québécois et la construction de la paternité dans l'après-guerre, 1945-1960", Revue d'histoire de l'Amérique française 57,4 (printemps 2004): 535-566; Michelle Roy, L'évolution de la figure paternelle dans les publicités du journal sherbrookois, La Tribune, au XXe siècle, mémoire de maîtrise (histoire), Université de Sherbrooke, 2007; Guillaume Saudrais, Maris et pères devant les tribunaux civils québécois, 1900-1920, mémoire de maîtrise (histoire), Université de Sherbrooke, 2010. Voir aussi Marie-Aimée Cliche, "Évolution du rôle du père au Québec du XVII ${ }^{\mathrm{e}}$ au XX ${ }^{\mathrm{e}}$ siècle», dans La paternité aujourd'hui "Bilan et nouvelles recherches, Actes du colloque du $66^{\mathrm{e}}$ Congrès de l'ACFAS, tenu à Québec le 12 mai 1998, Montréal, CLSC Côte-des-Neiges, Centre affilié universitaire, 5-12, consulté en ligne le 26 novembre 2015 au www.sherpa-recherche.com/ wp-content/uploads/2013/10/La_paternite_aujourdhui.pdf.

10. Voir Julie Néron et Camil Girard, «Vie quotidienne et rapport des genres. Mutation des espaces privé et public ", dans Camil Girard et Gervais Tremblay (dir.), Le Grand-Brûlé. Récits de vie et histoire d'un village au Québec, Laterrière, Saguenay, 1900-1960 (Québec, Les Presses de l’Université Laval, 2004),55-76; Catherine Cournoyer, «Les accidents impliquant des enfants et l'attitude envers l'enfance à Montréal (1900-1945)", mémoire de maîtrise (histoire), Université de Montréal, 1999; Marie-Aimée Cliche, Maltraiter ou punir? La violence envers les enfants dans les familles québécoises, 1850-1969 (Montréal, Boréal, 2007); Michael Gauvreau, Les origines catholiques de la Révolution tranquille, trad. Richard Dubois (Montréal, Fides, 2008). 
de sources distincts et complémentaires ${ }^{11}$. S'inscrivant dans le cadre de cette réflexion, notre propos ici vise, dans un premier temps, à offrir une esquisse rapide du schéma en trois modèles successifs qui structure, selon notre lecture, l'historiographie sur les pères au Québec depuis le XIX ${ }^{\mathrm{e}}$ siècle. Par la suite, nous proposerons le concept des visages de la paternité comme nouvelle grille d'analyse qui favorise une interprétation plus riche, plus nuancée et moins linéaire de cette histoire. Au final, nous puiserons dans deux corpus de sources, à savoir les récits de vie et les annonces publicitaires, pour démontrer à partir de quatre exemples à quel point ce concept des visages nous permet de faire avancer la discussion ${ }^{12}$. C'est dire en même temps qu'il est nécessaire et très possible de situer les pères québécois par rapport à une gamme de rôles, d'attentes et de responsabilités dynamique dans le temps, variable dans l'espace social ou géographique et beaucoup plus vaste et complexe que généralement reconnue.

\section{DU POURVOYEUR AU NOUVEAU PÈRE : UN SCHÉMA EN TROIS MODÈLES}

Dans notre lecture de l'historiographie pertinente, nous constatons la force, voire l'omniprésence, d'une mise en récit particulière, parfois explicite et parfois implicite, mais toujours évolutive et généralement structurée autour de trois modèles paternels qui se succèdent depuis la deuxième moitié du XIX ${ }^{e}$ siècle $^{13}$. Influencée notamment par l'abondante historiographie britannique, américaine et canadienne dans ce domaine ${ }^{14}$ et, dans

11. Dans le cadre d'un projet subventionné par le CRSH (Pères et paternité au Québec, 1900-1960), nous avons commencé en 2006 à constituer une banque de données, composée maintenant de plus d'un millier de fiches en format Filemaker et divisée en quatre corpus distincts: a) Expériences et récits de vie; b) Représentations normatives et médiatiques; c) Représentations littéraires; d) Lois et jurisprudence.

12. Comme toute source historique, celles-ci ont des avantages et des limites. Dans les récits de vie, l'expérience paternelle est évoquée la plupart du temps par le biais de souvenirs d'enfance et très rarement du point de vue du père lui-même. Dans une annonce publicitaire, une symbolique idéalisée (familiale ou autre) est utilisée pour vendre un produit sur le marché commercial. Notre analyse prendra donc très souvent des représentations de pères québécois comme point de départ, sans que le vécu de ces derniers ne soit évacué. En fait, ces deux catégories de sources ont une valeur inégale sur ce dernier point, les récits de vie étant plus aptes à nous livrer des renseignements sur de vrais hommes ayant vécu la paternité dans la période qui nous intéresse. Or, notre objectif étant d'offrir un aperçu de la richesse potentielle d'une grille d'analyse, celle des visages de la paternité, à partir de quelques exemples tirés d'un corpus documentaire beaucoup plus vaste, ces limites et ce déséquilibre ne devraient pas nous préoccuper outre mesure.

13. Pour une analyse plus poussée de cette historiographie, voir Peter Gossage, "Au nom du père? Rethinking the History of Fatherhood in Quebec ", American Review of Canadian Studies, 44, 1 (printemps 2014): 49-67.

14. Voir Ibid., pour un survol qui vise justement à mettre la production québécoise dans son contexte international. Parmi les titres importants, mentionnons Robert L. Griswold, Fatherhood in America: A History (New York, Basic Books, 1993); Ralph LaRossa, The Modernization of Fatherhood: A Social and Political History (Chicago, University of Chicago Press, 1997); Margaret Marsh, "Suburban Men and Masculine Domesticity, 1870-1915 », American Quarterly, 40 (1988): 165-186; et John Tosh, "Authority and 
une moindre mesure, par deux synthèses françaises parues vers la fin des années $1980^{15}$, cette narration, que nous présenterons ici sous forme très schématisée, voire caricaturale, pourrait ressembler à ceci :

$\mathrm{Au} \mathrm{XIX}^{\mathrm{e}}$ siècle et au début du $\mathrm{XX}^{\mathrm{e}}$ siècle, le modèle du père pourvoyeur domine. Ce père-là, souvent qualifié de traditionnel, s'occupe essentiellement de la survie et du bien-être de sa famille sur le plan matériel. Plutôt réservé, il s'exprime peu en ce qui concerne ses sentiments profonds. Préoccupé par son travail, que ce soit à la ferme, à l'usine, au bureau ou ailleurs, il laisse à sa conjointe ("ménagère» ou "reine du foyer») les soins et l'éducation des enfants, sauf ceux (généralement les fils) qui sont en mesure de l'aider dans son travail. Sans nécessairement être autoritaire, ce père est investi, par la culture et par le Code civil, d'une grande autorité. Il est donc responsable de la discipline et possède le dernier mot sur toute décision familiale importante. C'est justement ce modèle de «l'homme pourvoyeur" qui se dégage, par exemple, des entrevues effectuées avec des Saguenéens par l'équipe de Camil Girard et Gervais Tremblay. Qu'il soit «agriculteur, travailleur forestier, ou professionnel», la responsabilité principale du père au village de Laterrière est « de faire vivre sa femme et ses enfants ${ }^{16}$.

Dans le courant du $\mathrm{XX}^{\mathrm{e}}$ siècle, le modèle plus moderne et plus souple du père éducateur se développe. Cet homme-là s'occupe davantage de ses enfants - notamment de ses fils, surtout à partir de l'âge de 10-12 ans - et tout particulièrement de leur éducation intellectuelle et morale. Il donne un bon exemple de rectitude, de sobriété et surtout de responsabilité. Il conserve la fonction de gagne-pain familial, mais il est beaucoup plus présent sur le plan émotif. Il passe son temps de loisirs à faire des activités familiales: le sport, les vacances, les fêtes, etc. Ce sont les grandes mutations sociales du $\mathrm{XX}^{\mathrm{e}}$ siècle qui permettent son émergence, dont la montée d'une classe

Nurture in Middle-Class Fatherhood: The Case of Early and Mid-Victorian England», Gender \& History, 8, 1 (1996): 48-64. Pour le Canada, voir Cynthia Comacchio, “"A Postscript for Father" : Defining a New Fatherhood in Interwar Canada ", Canadian Historical Review 78, 3 (1997): 385-408; Christopher Dummitt, "Finding a Place for Father: Selling the Barbecue in Postwar Canada», Revue de la Société historique du Canada, 9 (1998): 209-223 ; ainsi que les nombreux articles et chapitres de Robert Rutherdale ; voir par exemple, «Fatherhood and Masculine Domesticity During the Baby Boom: Consumption and Leisure in Advertising and Life Stories", dans Lori Chambers et Edgar-André Montigny (dir.), Family Matters: Papers in Post-Confederation Canadian Family History, (Toronto, Canadian Scholars' Press, 1998) : 309-333; "New "Faces" for Fathers: Memory, Life Writing, and Fathers as Providers in the Postwar Consumer Era », dans Magda Fahrni et Robert Rutherdale (dir.), Creating Postwar Canada: Community, Diversity, and Dissent, 1945-1975 (Vancouver, University of British Columbia Press, 2008) : 241-267.

15. Yvonne Knibiehler, Les pères aussi ont une histoire (Paris, Hachette, 1987); Jean Delumeau et Daniel Roche (dir.), Histoire des pères et de la paternité (Paris, Larousse, 1990).

16. J. Néron et C. Girard, «Vie quotidienne et rapport des genres», loc. cit., 61-67. 
Figure I

Le père idéal dans les années 1950

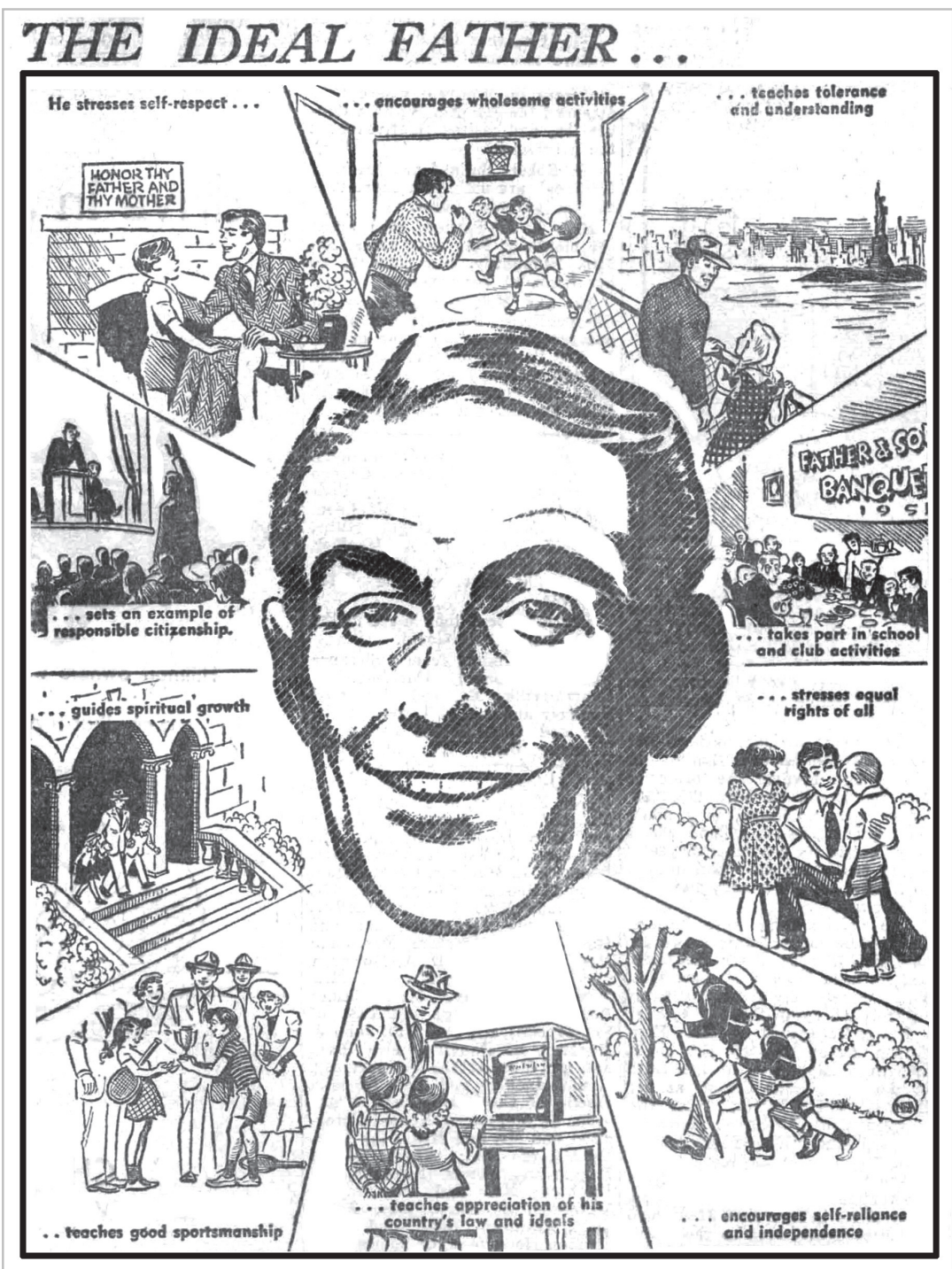

Il s'agit d'un homme qui, dans ses relations avec ses enfants, «inspire l'estime de soi, encourage les activités saines, enseigne la tolérance et la compréhension, donne un exemple de citoyenneté responsable, encadre la croissance spirituelle, enseigne l'esprit sportif, encourage une appréciation des lois et valeurs de sa patrie, inspire l'autonomie et l'indépendance, fait la promotion des droits égaux pour tous et s'implique dans les activités à l'école et dans les associations. » Source: Sherbrooke Daily Record, le 12 juin 1951, 11. 
moyenne urbaine aux revenus supérieurs et aux temps libres plus abondants, sans oublier le déclin de la taille des familles. On a souvent recours au concept de domesticité masculine, développé par Margaret Marsh, pour rendre compte de cette nouvelle réalité paternelle, quand ce n'est pas à la paternité nouvelle de Robert Griswold ou à la paternité moderne de Ralph LaRossa ${ }^{17}$. Ce rôle paternel élargi sur les plans éducatif et récréatif est évoqué, par ailleurs, dans le dessin éditorial de 1951 reproduit à la figure 1, comme dans la plupart des matériaux (publicitaires, notamment) qui entourent la fête des Pères: nouvelle "tradition» familiale inventée aux États-Unis au début du siècle, et traitée par LaRossa comme un rituel de mise en scène d'une paternité modernisée, notamment durant l'entre-deux-guerres ${ }^{18}$.

Vers la fin du $\mathrm{XX}^{\mathrm{e}}$ siècle, le nouveau père fait son entrée en scène, appelé par les grandes mutations sociales et familiales de l'époque récente, dont les gains réalisés par le mouvement féministe, la transformation des mours en matière de sexualité et de reproduction, la libéralisation de l'accès au divorce, la généralisation des unions libres et la chute dramatique de la natalité. Cette nouvelle parentalité masculine prend différentes formes, en fonction de la diversité croissante des structures familiales. Dans le cas de la famille nucléaire dite "traditionnelle» (couple hétérosexuel vivant avec ses enfants biologiques), le nouveau père partage de manière plus égale les soins et l'éducation des enfants avec sa conjointe qui, elle, assume sa part du rôle de pourvoyeur dans le cadre d'un ménage à double revenu. Ce nouveau père tient à son rôle de parent, accompagnant sa conjointe à travers la grossesse et l'accouchement et s'occupant pleinement de ses enfants dès la petite enfance. Pas particulièrement disciplinaire, le contenu émotif de sa relation parentale est riche et valorisant, tant pour lui-même que pour ses enfants. C'est le cas, par ailleurs, de milliers de pères qui, à la suite d'une rupture de couple, n'auront pas la garde des enfants mais tiennent fermement au maintien d'une proximité affective et à jouer un rôle important

17. R. Griswold, Fatherhood in America, op. cit.; R. LaRossa, The Modernization of Fatherhood, op. cit.; M. Marsh, "Suburban Men and Masculine Domesticity», op. cit. Dans les travaux de Vincent Duhaime, c'est justement cette nouvelle conception moins rigide et plus engagée des responsabilités et des rôles paternels que l'auteur cherche à situer dans l'immédiat après-guerre. Duhaime identifie une tension importante entre le nouveau modèle du "père éducateur» et les conceptions traditionnelles du père et du masculin au Québec, dont celles véhiculées par le mouvement familial catholique entre 1945 et 1965 . Voir V. Duhaime, $\mathrm{La}$ construction du père québécois, op. cit., et Duhaime, "Les pères ont ici leur devoir" ", loc. cit.

18. Selon l'hypothèse de LaRossa, la fête des Pères "... was the ritual that embodied America’s New Fatherhood. Through the ceremony of giving and receiving Father's Day gifts, families could "do" or "enact" the modernization of fatherhood", R. La Rossa, The Modernization of Fatherhood, op. cit., 189. Pour notre analyse historique de la fête des Pères au Québec, voir Peter Gossage, "Celebrating the Family Man: From Father's Day to La Fête des Pères, 1910-1960 », dans Peter Gossage et Robert Rutherdale (dir.), Masculine Histories in the Making: Emerging Themes in Canada (Vancouver, UBC Press, à paraître). 
dans l'éducation de ces derniers. Et c'est sans parler des milliers d'hommes qui vivent leur parentalité autrement de nos jours, que ce soit comme chef d'une famille monoparentale, comme membre d'un couple homoparental ou comme père substitut ou adoptif dans le cadre d'une famille recomposée ${ }^{19}$.

Voilà donc le schéma d'une transformation séculaire en trois temps dont la valeur descriptive est considérable, en raison notamment de sa simplicité et de sa cohérence. Jusqu'à présent, les historiens s'en sont bien servis, surtout pour analyser la période d'avant 1970, faisant ressortir le passage (mais aussi la longue cohabitation) entre le premier modèle et le deuxième, c'est-à-dire la diffusion graduelle, face aux résistances et aux ambivalences importantes, d'une manière plus souple, plus engagée et plus accessible d'être père au Québec ${ }^{20}$. Mais malgré tout, comme la plupart des schémas globalisants, celui-ci comporte des inconvénients. Serait-il, par exemple, un peu trop sommaire et trop linéaire pour rendre compte de la complexité et de la diversité des expériences, des représentations et des idéologies de la paternité québécoise? Aurait-il tendance aussi à mettre de l'avant un portrait un peu trop homogène de la parentalité masculine, en nous proposant une catégorie "père» qui réfère essentiellement (ou implicitement) aux hommes blancs, chrétiens, non issus de l'immigration, hétérosexuels et dans la force de l'âge puisqu'on les associe à leur rôle de pourvoyeur ${ }^{21}$ ? L'avertissement émis récemment par l'historienne britannique Laura King, par ailleurs, nous paraît des plus pertinents:

We must be mindful here of the recurrent idea of change in fatherhood that can be found throughout modern history; as [British psychologist] Charlie Lewis noted, the

19. Dans son livre sur la paternité contemporaine, Lori Saint-Martin évoque cette diversité, soulignant entre autres des ambivalences et des tensions qui en découlent et qui font poser la question, "Les "nouveaux pères" existent-ils?»; L. Saint-Martin, Au-delà du nom, op. cit., 42. C’est là une preuve sans contredit de la force, dans le discours contemporain, de l'idéal du nouveau père, sans que l'on puisse évoquer un consensus sur la réalité.

20. Les travaux de Vincent Dumaine et de Guillaume Saudrais, notamment, vont dans ce sens. Voir V. Duhaime, La construction du père québécois, op. cit. et V. Duhaime, "Les pères ont ici leur devoir" ", loc cit.; G. Saudrais, Maris et pères devant les tribunaux, op. cit.. Pour sa part, Michelle Roy pousse son analyse jusqu'aux années 1990 et retrace de nombreuses représentations du «nouveau père» dans ses sources; voir M. Roy, L'évolution de la figure paternelle, op. cit. Sur le plan conceptuel, par ailleurs, cette auteure insiste plus explicitement que d'autres sur le schéma évoqué ici d'une évolution en trois étapes des modèles paternels au $\mathrm{XX}^{\mathrm{e}}$ siècle.

21. Nous tenons à remercier Marie-Laurence B. Beaumier d'avoir partagé cette observation avec nous. Sommes-nous, demande-t-elle, en face d'une «représentation hégémonique» de la paternité, pour paraphraser R. W. Connell? Voir R. W. Connell et J. W. Messerschmidt, «Hegemonic Masculinity: Rethinking the Concept", Gender \& Society, 19 (décembre 2005): 829-859. 
idea that men have recently become more involved in family life " is as old and perhaps as prominent as patriarchy" ${ }^{22}$.

Sans vouloir jeter le bébé avec l'eau du bain (pour se servir d’une métaphore parentale), nous sommes donc à la recherche d'une grille conceptuelle plus souple, permettant des analyses plus fines et diversifiées de la parentalité masculine durant le $\mathrm{XX}^{\mathrm{e}}$ siècle au Québec.

\section{LES VISAGES DE LA PATERNITÉ}

C'est dans cette optique que nous avançons ici la grille des visages de la paternité, proposée il y a plus de trente ans par John Demos et renouvelée récemment par Robert Rutherdale. Dans un texte intitulé The Changing Faces of Fatherhood, Demos offre, en 1982, un survol des différentes manières de vivre et de comprendre le rôle paternel aux États-Unis de l'époque coloniale jusqu'aux années 1970. C'est à cette longue liste de façons d'être père, compilées et soulignées tout au long de son texte, que réfère l'auteur lorsqu'il insiste sur les visages multiples et changeants du père à travers l'histoire. Pour l'époque coloniale, les visages paternels sont étonnamment nombreux et variés: «father as pedagogue ... benefactor ... controller ... moral overseer, voire psychologist ... caregiver ... example ... companion».

$\mathrm{Au} \mathrm{XIX}^{\mathrm{e}}$ siècle, certains visages paternels prennent du recul et laissent la place à d'autres (father as discussion leader... disciplinarian). La nouvelle place prise par le visage du père pourvoyeur est plus significative encore (father as provider):

Now, being fully a father meant being separated from one's children for a considerable part of every working day [...]. And, at the same time, father's intrinsic connection to all that lay outside home gave him a special status within $i t^{23}$.

Dans le courant du $\mathrm{XX}^{\mathrm{e}}$ siècle, le visage du père comme copain (father as chum) commence à prendre de la place, surtout du point de vue des fils avec lesquels les hommes partagent des activités sportives et récréatives. La donne change de nouveau à la suite des mutations sociales des années 1960 et 1970, surtout avec la montée des divorces qui fait en sorte que les pères portent encore de nouveaux visages, dont certains positifs (les single

22. Laura King, Family Men: Fatherhood and Masculinity in Britain, 1914-1960 (Oxford, Oxford University Press, 2015), 3.

23. John Demos, "The Changing Faces of Fatherhood", dans Past, Present and Personal: The Family and the Life Course in American History (New York, Oxford University Press, 1986), 52. Il s'agit d'une réimpression de "The Changing Faces of Fatherhood: A New Exploration in American Family History" dans Stanley H. Cath, Alan R. Gurwitt et John M. Ross (dir.), Father and Child: Developmental and Clinical Perspectives, (Boston, Little Brown \& Co., 1982), 425-445. 
dads dévoués et courageux) et certains tout le contraire, comme les deadbeat dads: ceux qui abandonnent toute responsabilité envers leurs enfants pour poursuivre des objectifs plus égoïstes ${ }^{24}$.

Presque trente ans plus tard, Robert Rutherdale publie un texte inspiré des idées de Demos dans un ouvrage collectif édité en Angleterre sur l'histoire du masculin ${ }^{25}$. Trois visages de la paternité canadienne y sont mis de l'avant: a) les pères tyranniques, hyper-autoritaires; b) les pères éducateurs, pédagogues, soucieux de la formation de leurs enfants; c) les "workaholics»-ceux qui sont absents sur le plan émotif parce qu'ils sont toujours au boulot, pour lesquels le travail devient une obsession. Tout cela est dressé avec en arrière-plan une norme ou une idéologie particulière de la paternité - celle de la "responsible family manhood»-qui serait de plus en plus dominante dans ce contexte particulier, à savoir le Canada anglais à l'époque des "Trente Glorieuses» (1945-1975). Rutherdale explique ainsi sa manière de concevoir le concept des visages de la paternité, et surtout sa valeur comme cadre conceptuel pour une analyse des itinéraires paternels individuels:

From intimate family relations to their public roles in work and local communitybased leisure, fathers" varied masculinities emerge in identifiable categories - as "faces of fatherhoods" - but only as outcomes of their parenthood that were relationally constructed over the life course. Fathers exercised their responsibilities through a variety of potential behaviours connected to their basic function of provider and parent. Fatherhood's many potential faces, as John Demos suggested before work on the subject began its fruitful escalation, are a useful way to conceptualise and categorise their masculinity as family men ${ }^{26}$.

Si nous prenons la peine de présenter cette grille des visages de la paternité, c'est que nous la considérons bien adaptée à l'analyse des multiples manières de comprendre et de vivre l'expérience paternelle au Québec à travers le temps. Conçus à la manière de Rutherdale, comme un éventail de comportements masculins potentiels liés aux fonctions de pourvoyeur et de parent, quels sont donc les visages de la paternité québécoise que l'on peut identifier dans les documents historiques? Comment les hommes comprennent-ils et construisent-ils leur identité masculine à travers leur

\footnotetext{
24. Ibid.

25. Robert Rutherdale, "Three Faces of Fatherhood as a Masculine Category: Tyrants, Teachers, and Workaholics as "Responsible Family Men” During Canada’s Baby Boom ", dans John H. Arnold et Sean Brady (dir.), What is Masculinity? Historical Dynamics from Antiquity to the Contemporary World, (Basingstoke, Royaume-Uni, Palgrave Macmillan, 2011), 323-348.

26. Ibid., 325.
} 
expérience et leur itinéraire de père de famille? Y a-t-il, par ailleurs, un agencement de ces visages que l'on peut appréhender dans la diachronie, en fonction d'une conjoncture particulière (guerre, crise ou période de prospérité) ou bien dans le cadre d'une analyse synchronique, en comparant les différentes composantes de la société québécoise?

Sans répondre entièrement à ces questions, nous proposons ici quatre visages de la paternité québécoise identifiés dans deux catégories de source, à savoir les récits de vie et les annonces publicitaires ${ }^{27}$. Nous les appelons le père spirituel, le père disciplinaire, le père prévoyant et le père sportif et nous les présenterons ici, à titre d'exemples, dans l'idée de démontrer la valeur heuristique de cette manière de concevoir la parentalité masculine dans le cas du Québec durant les six premières décennies du XX $\mathrm{X}^{\mathrm{e}}$ siècle. Sans être entièrement aléatoire, notre choix de ces quatre visages pour les fins actuelles s'est fait par le biais d'un processus plus personnel que systématique, en fonction de l'intérêt plutôt que de l'importance de chaque exemple. Nous n'avancerons pas de thèse quant à la prédominance relative de l'un ou l'autre de ces visages, que ce soit dans nos sources ou dans la société qui les a produites. Mais comme on le verra, une analyse des interactions entre ces différentes façons de comprendre et de vivre la parentalité masculine, choisies parmi bien d'autres pour les fins de la démonstration, reste tout à fait possible et pertinente. Et c'est là toute la richesse et le potentiel d'une histoire des pères libérée de ces trois modèles plus ou moins figés et réinscrite dans un cadre dynamique plus souple et polyvalent.

\section{Le père spirituel}

Toute analyse de la paternité au Québec doit tenir compte d'une dimension fondamentale de celle-ci, à savoir le pouvoir ou l'autorité. La puissance paternelle reste un concept fondamental en droit civil et la zone des tensions et des contradictions entre cette autorité et celle des instances étatiques reste un terrain d'enquête très fertile ${ }^{28}$. Tout en reconnaissant

27. Pour les récits de vie, l'analyse repose essentiellement sur quelque 76 pages de témoignages transcrits à partir d'ouvrages autobiographiques écrits par 23 Québécois nés entre 1888 et 1941. Pour la plupart, ces individus ( 15 hommes et 8 femmes) évoquent des souvenirs d'enfance et la relation qu'ils ont vécue avec leur père. Deux d'entre eux seulement (Gérard Parizeau, 1899-1994 et Réjean Lafleur, 1928-1992) évoquent en détail leur propre expérience parentale. Pour les annonces publicitaires, nous puisons dans notre corpus de 286 annonces, publiées entre 1900 et 1960, tirées surtout de deux quotidiens (Le Soleil et The Sherbrooke Daily Record). Notons que le dépouillement a porté sur les mois de juin et de décembre aux intervalles de cinq ans.

28. Les travaux de Dominique Marshall sont particulièrement importants pour toute analyse des lignes de force entre le pouvoir familial/patriarcal et le pouvoir étatique; voir notamment D. Marshall, Aux origines sociales de l'État-providence (Montréal, Presses de l'Université de Montréal, 1998). Pour une approche plus judiciaire, mettant en opposition le pouvoir disciplinaire du père et celui de l'État, voir 
la valeur de l'approche sociojuridique ${ }^{29}$, nous avons choisi de mettre l'accent ici sur la composante spirituelle de cette autorité paternelle dite traditionnelle. Rappelons qu'à une certaine époque, dans les milieux chrétiens, le père de famille incarnait l'autorité d'un Dieu dont la forme est la sienne; il était donc le chef spirituel de sa famille. Yvonne Knibiehler, dans l'une des premières monographies portant sur l'histoire des pères, consacre tout un chapitre à cette conception du père spirituel au sein des familles chrétiennes, et ce, dès le Moyen Âge:

Le père chrétien est donc le seul chef de la famille et seul maître de ses enfants. Son autorité sur eux n'est jamais discutée. Sa sévérité est légitime, car les enfants, dépourvus de raison, sont en outre pétris de mauvais instincts. [... Mais les prêtres] rappellent volontiers au père le principe essentiel qui doit guider sa conduite: ses enfants sont d'abord enfants de Dieu. Ils n'ont par eux-mêmes aucun «droit naturel» mais ils appartiennent au Créateur. Les droits de Dieu transforment en devoirs le pouvoir du père ${ }^{30}$.

Pour revenir au Québec, il nous paraît assez clair que cette longue tradition chrétienne - celle qui fusionnerait les instances d'autorité divine et paternelle - persiste, sous différentes formes, jusqu'à l'époque contemporaine. Le vocabulaire du pouvoir au sein de l'Église catholique est fondé notamment sur des métaphores familiales et patriarcales (Notre Père qui est aux Cieux; le Saint-Père à Rome; le mot «Père " comme titre honorifique mérité par tout membre du clergé, etc.), ce qui fournit un rappel constant et éclairant de cette réalité. Il existe par ailleurs une cérémonie dans la culture canadienne-française qui symbolise cette relation d'autorité et de soumission spirituelles. Il s'agit bien entendu de la bénédiction paternelle du jour de l'An: rituel en perte de vitesse au XX $\mathrm{X}^{\mathrm{e}}$ siècle que l'on doit certainement lire comme performance (et éventuellement comme vestige) de l'autorité patriarcale ancienne ${ }^{31}$.

Il existe plusieurs descriptions de cette cérémonie, souvent assez nostalgiques, dans les récits de vie que nous avons consultés (voir aussi la figure 2).

M.-A. Cliche, Maltraîter ou punir?, op. cit. et Tamara Myers, Caught: Montreal's Modern Girls and the Law, 1869-1945 (Toronto, University of Toronto Press, 2006).

29. Le mémoire de maîtrise de Guillaume Saudrais en fournit un bel exemple; voir G. Saudrais, Maris et pères devant les tribunaux civils, op. cit.. Nous poursuivons l'analyse avec cet angle d'approche dans Peter Gossage, "On Dads and Damages: Looking for the "Priceless Child" and the "Manly Modern" in Quebec's Civil Courts, 1921-1960", Histoire sociale/Social History, 49,100 (2016): 603-623.

30. Y. Knibiehler, Les pères aussi ont une histoire, op. cit., 55.

31. Vincent Duhaime cite un article de la revue catholique Foyer rural publié en 1946, où l'auteur plaide pour «la survie de la bénédiction paternelle, tradition ancestrale [qui] rappelle que le père est "le chef naturel de la famille, qu'il reçoit son autorité de Dieu même”"; V. Duhaime, "Les pères ici ont leur devoir" ", loc. cit., 554. 
Figure 2

\section{La Bénédiction paternelle au Jour de l'An}

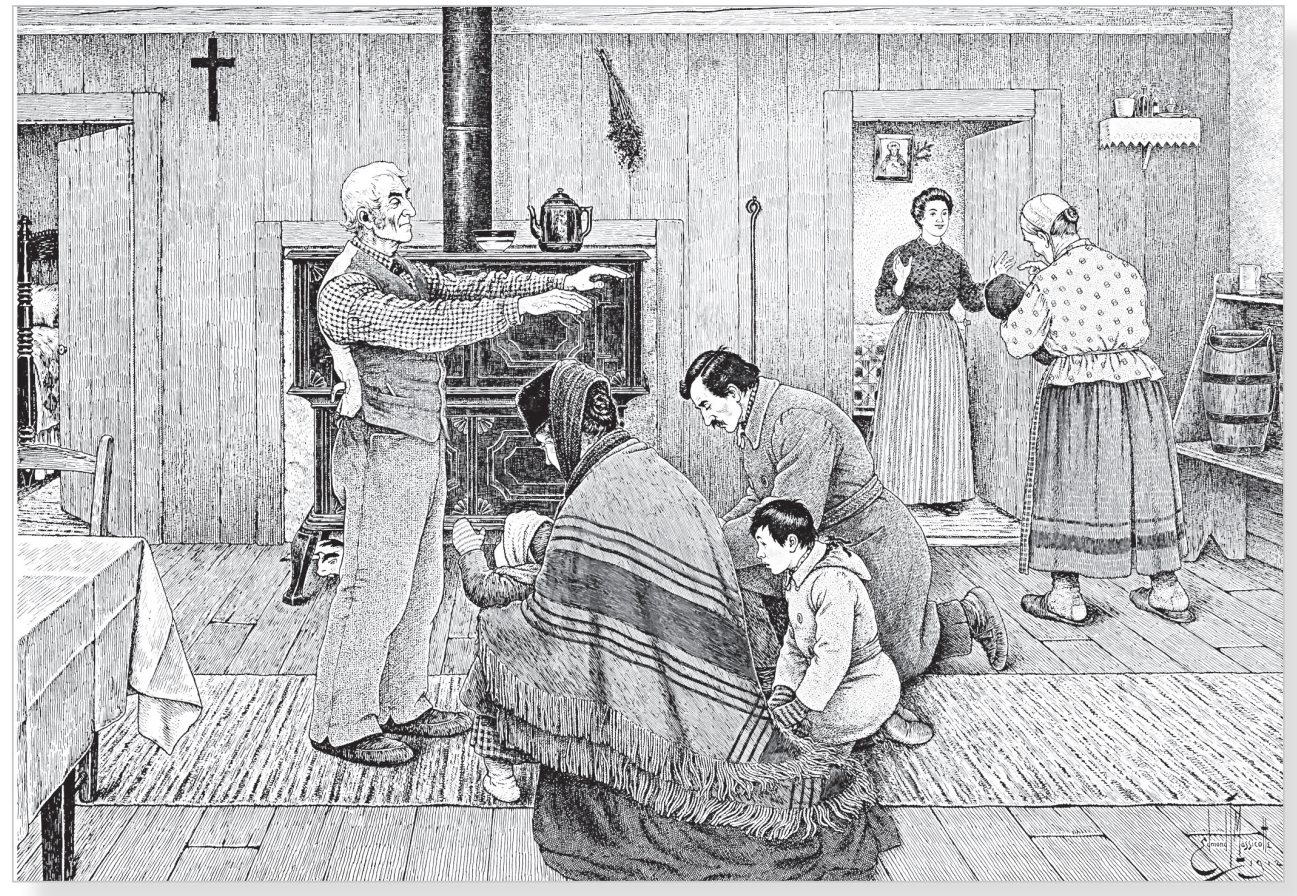

Edmond-Joseph Massicotte, La bénédiction du jour de l'An, 1912, photogravure et rehauts d'aquarelle, photographié par Denis Legendre.

Source: Collection du Musée national des beaux-arts du Québec, reproduit avec permission.

Celle de Simonne Monet-Chartrand (1919-1993) nous parait assez typique. Nous sommes le 2 janvier 1931 : «Hier, comme à l’accoutumée, mon frère aîné a demandé en notre nom la bénédiction paternelle. Papa était bien ému. Il nous a signé le front, nous a embrassés puis nous a dit: "Que le bon Dieu, que je représente, vous bénisse et vous rende heureux" ${ }^{\prime 2}$ ». Renée Morin, quant à elle, raconte la disparition de cette tradition au sein de sa famille et se questionne sur les sentiments de son père, le notaire Victor Morin (1865-1960), face à cette transformation, signe des temps modernes:

32. Simonne Monet-Chartrand, Ma vie comme rivière: Récit autobiographique. Tome 1, 1919-1942 (Montréal, Les éditions du Remue-ménage, 1981), 93. 
La bénédiction paternelle était une cérémonie à la fois simple et solennelle où les enfants à genoux recevaient sur leur tête l'imposition des mains du père qui priait Dieu de les combler de ses grâces. Quand les aînés commencèrent à vieillir, cette cérémonie leur semblait gênante; ils trouvaient de bonnes excuses pour ne pas être à la maison le moment venu. [...] Enfin, il y eut un premier Jour de l'An où mon père, assis à sa table de travail, dans la bibliothèque attendait, comme chaque année, un peu avant l'heure du midi, la venue de quelques enfants ou petits-enfants et personne ne se présenta. Je me demande ce qu'il ressentit ce jour-là? Il admit sans doute que les temps avaient changé. En tout cas, il n'en souffla mot ${ }^{33}$.

Pour sa part, l'écrivaine Claire Martin (1914-2014) dévoile sa haine pour un père carrément tyrannique dans son extraordinaire récit autobiographique, qualifié récemment par Patricia Smart comme «le premier ouvrage explicitement féministe de la littérature québécoise ${ }^{34}$ ». Martin raconte la bénédiction paternelle dans sa famille comme une sorte d'épreuve de force entre cet homme autoritaire - qui insiste de manière arbitraire sur une bénédiction individuelle pour chaque enfant - et ses enfants qui s'empressent pour éviter une telle entrevue privée:

Le lendemain matin, il fallait s'exécuter. Seulement, quand le plus courageux d'entre nous voulut rejoindre mon père dans le cabinet de travail, où il venait de le voir entrer, il trouva la pièce vide. Mon père était ressorti par l'autre porte, avait traversé rapidement l'antichambre et se trouvait, maintenant dans le salon où le malheureux candidat à la bénédiction se précipita. [...] Je ne sais pas bien comment expliquer ces allées et venues si ce n’est par le plaisir sadique qu'il prenait à nous voir tournoyer d'une pièce à l'autre. L'explication de la bénédiction-solo était plus évidente. C’était le moment rêvé pour faire la revue générale des engueulades de l’année ${ }^{35}$.

\section{Le père disciplinaire}

Le père disciplinaire, juge de dernière instance et auteur des punitions corporelles - «Attends que ton père arrive», disaient certaines mères d'autrefois $^{36}$ - est un autre visage paternel que l'on peut déceler dans cet ensemble de témoignages. Mais quelle distance proposer entre le père violent de Claire Martin et d'autres hommes qui interviennent pour «corriger» leurs

33. Renée Morin, Un bourgeois d’une époque révolue: Victor Morin, notaire (1865-1960) (Montréal, Les Éditions du Jour, 1967), 134-135.

34. P. Smart, De Marie de L'Incarnation à Nelly Arcan..., op. cit., 286.

35. Claire Martin, Dans un gant de fer, Édition critique par Patricia Smart (Montréal, Presses de l'Université de Montréal, [1965-1966], 2005), 2 e Partie, «La joue droite», 284-285.

36. L. Saint-Martin, Au-delà du nom..., op. cit., 9. 
enfants avec une mesure de force physique jugée raisonnable? Il s'agit là d'une question dont les multiples facettes ont déjà été étudiées en profondeur par Marie-Aimée Cliche. Dans la période qui nous préoccupe, comme Cliche le démontre, les débats se prolongent entre «la méthode forte» - où figurent la baguette, le fouet et un moralisme rigide inspiré de la Bible - et des méthodes plus libérales favorisant l'épanouissement de l'enfant et fondées sur de nouvelles «connaissance[s] scientifique[s] de la psychologie de l'enfant, considéré comme l'agent premier de son éducation ${ }^{37}$. Sans qu'il n'y ait de progression nette ou linéaire, par ailleurs, la philosophie éducative rigide du début du siècle, qui conçoit les punitions corporelles comme «un droit[,] voire un devoir parental ${ }^{38}$ ", cède graduellement sa place à une approche plus libérale, fondée sur l'amour et sur de nouvelles connaissances en psychologie de l'enfance.

Si les experts restent divisés pendant de longues décennies sur la place des «taloches» et des «fessés» dans une philosophie éducative moderne, il parait évident que les pères de l'époque le sont aussi. Car en vertu du Code civil, et ce jusqu'en 1977, c'est en premier lieu le père qui possède ce «droit de correction modérée et raisonnable» sur son enfant mineur, même si la mère peut assumer cette responsabilité «à son défaut» et si les parents peuvent la déléguer "à ceux à qui l'éducation de cet enfant a été confiée »" Mais dans quelle mesure, de quelle manière et dans quelles circonstances les hommes choisissent-ils de la mettre en application: de porter le visage, autrement dit, du père disciplinaire? Il y a forcément toute une gamme de réponses à cette question, allant des plus sévères aux plus indulgentes. Nous pouvons sans aucune hésitation, par exemple, situer le père tyrannique de Claire Martin du côté extrême de l'échelle. Voici sa manière à elle de décrire le style de corrections auquel cet homme violent se livrait régulièrement, et ce, dans un milieu bourgeois des années 1920:

Quelle punition? Mais toujours la même. Cela commençait par quelques gifles mais tout de suite des grosses, puis, l'entrain venant, cela se continuait par des coups de poing et, si l'entrain tenait, cela finissait par des coups de pied qui nous transbordaient d'une pièce à l'autre sur tous les parquets du rez-de-chaussée. Quand on songe qu'il mesurait six pieds, qu'il pesait deux cent trente livres, qu'il avait accompli dans sa jeunesse des exploits à la Jean Valin, dont toute l'île

37. M.-A. Cliche, Maltraiter ou punir..., op. cit.,163-64.

38. Ibid., 129.

39. Paul-André Crépeau et John E. C. Brierley, Code civil-Civil code, 1866-1980: Édition historique et critique - An Historical and Critical Edition (Montréal, Chambre des notaires du Québec - Société québécoise d'information juridique, 1981), Article 245 (1866-1977), 114. 
d'Anticosti - où il avait commencé sa carrière d'ingénieur - parlait encore, c'est quand même étonnant qu'il n'ait tué aucun de nous ${ }^{40}$.

À l'autre bout du spectre se trouve le père de Jeannette Vekeman-Masson (1899-1989), le journaliste belge Gustave Vekeman (1841-1916). Au Canada depuis 1882, ce dernier s'installe à Grosse-Île en 1906 avec sa jeune famille dans un poste de traducteur/interprète auprès des immigrants européens ${ }^{41}$. Vekeman avait 58 ans à la naissance de Jeannette, l'aînée des quatre enfants de son deuxième lit, au tournant du siècle. Vers la fin de sa vie, Jeannette raconte dans ses Mémoires un épisode où elle a été chargée de veiller sur ses trois petits frères mais ne réussit pas à maintenir la paix. Son père la tient responsable du bruyant échange d'insultes qui s'ensuit et intervient pour envoyer Jeannette dans sa chambre sans dessert. À la suite de la narration de ces événements, elle enchaîne en faisant ressortir le but de son anecdote: «Papa ne nous a jamais frappés, mais il avait le tour de nous faire comprendre sans commandement ${ }^{42}$.»

Entre ces deux pôles se situe Gérard Parizeau (1899-1994) qui, en rappelant ses propres méthodes de discipline auprès de ses deux fils, avoue qu’il croyait à la correction corporelle «modérée » et seulement "avant un certain âge» :

Autant nous détestions la gifle qui vient s'écraser sur la figure de l'enfant et l'humilier, autant nous pensions que la région fessière était l'endroit idéal pour rappeler l'esprit d'obéissance à ces petits êtres sauvages, indomptés, que sont les gosses en bas âge. La main nue et, parfois, le dos de la brosse à cheveux étaient nos armes de combat. Nous n'en abusions pas, mais il y avait des moments où l'application de l'une ou de l'autre avec la vigueur voulue avait l'effet recherché, pour un temps. Quoi qu'en pensent certains psychologues, psychiatres ou pédiatres, Germaine et moi, sans être partisans de la manière forte, nous croyions qu'avant un certain âge, la correction corporelle modérée, mais ferme, était excellente ${ }^{43}$.

40. C. Martin, Dans un gant de fer, $1^{\text {re }}$ partie, «La joue gauche», op. cit., 97.

41. Voir Bernard Vekeman avec la collaboration de Rose Masson Dompierre, Ouvrier de la plume: Gustave Vekeman, Ouvrier, imprimeur, journaliste et écrivain belge, Belgique 1841-1882, Canada 1882-1916 (Ottawa, Pierrette et Hélène Vekeman, 2008), 333.

42. Jeannette Vekeman-Masson, Grand-maman raconte La Grosse Île (Ottawa, Corporation pour la mise en valeur de Grosse-île inc., 2001), 118-119. Les souvenirs du journaliste et écrivain Paul Toupin (19171993), dont le père ne l'avait «jamais battu et je ne me souviens pas d'avoir jamais reçu une gifle», vont dans le même sens. Paul Toupin, Souvenirs pour demain (Ottawa, Le cercle du livre de France, 1960), 67.

43. Gérard Parizeau, Joies et deuils d'une famille bourgeoise, 1867-1961 (Trois-Rivières, Bien Public, 1973), 210. 
Parizeau prend ainsi ses distances par rapport aux conseils de certains experts modernes ("psychologues, psychiatres ou pédiatres») qui vont dans le sens d'un refus total de la force physique. Ses propos semblent correspondre à un consensus que Cliche situe dans les années 1950: les punitions corporelles modérées, généralement sous forme de tapes à main nue, sont admises mais réservées aux cas exceptionnels et jamais infligées sous le coup des nerfs ou de la colère.

\section{Le père prévoyant}

Homme d'affaires, écrivain, éducateur et fondateur de la revue Assurances, Gérard Parizeau nous offre un pont inattendu vers le troisième visage du père québécois proposé ici, celui-ci repéré essentiellement dans les annonces publicitaires. Il s'agit du père qui assume pleinement son rôle de pourvoyeur, tout en s'engageant dans une des dimensions importantes de la modernité occidentale du $\mathrm{XX}^{\mathrm{e}}$ siècle, à savoir la gestion du risque ${ }^{44}$. Nous l'appelons le père prévoyant, celui qui a le souci de préparer soigneusement l'avenir de ses enfants, et ce, même au-delà de sa propre mort. Rappelons que la prévoyance se situe dans cette gamme de valeurs individualistes associées à l'ordre libéral moderne, s'opposant d'un côté à l’imprévoyance dont les pères de la classe ouvrière sont si souvent accusés et de l'autre à ces valeurs plus anciennes, collectivistes et populaires que sont l'entraide, le mutualisme et la providence ${ }^{45}$.

$\mathrm{Au} \mathrm{XX}^{\mathrm{e}}$ siècle, la possibilité de gérer un risque en particulier, celui de laisser sa veuve et ses enfants dans un état d’indigence, constitue sans

44. Sur la gestion du risque comme élément de la modernité industrielle au début du $\mathrm{XX}^{\mathrm{e}}$ siècle, voir notamment Magda Fahrni " "La lutte contre l'accident" : Risque et accidents dans un contexte de modernité industrielle», dans David Niget et Martin Petitclerc (dir.), Pour une histoire du risque: Québec, France, Belgique (Québec, Presses de l'Université du Québec, 2012), 181-202. Sur le rapport entre modernité, risque et masculinité durant l'après-guerre en Colombie-britannique, voir Christopher Dummitt, The Manly Modern: Masculinity in Postwar Canada (Vancouver, UBC Press, 2007). Nous explorons nous-même une autre dimension de la gestion du risque chez les pères québécois au $\mathrm{XX}^{\mathrm{e}}$ siècle dans $\mathrm{P}$. Gossage, "On Dads and Damages... », loc. cit. Dans cette analyse d'un corpus d'articles de jurisprudence, l'un des enjeux principaux est la responsabilité juridique du père dans le cas où son enfant mineur est reconnu comme l'auteur d'un délit ayant causé des dommages physiques ou moraux à autrui. Jusqu'à quel point cet homme-là peut-il être jugé coupable d'avoir exercé un niveau de prévoyance paternelle insuffisant?

45. Voir Gabrielle Roy, Bonheur d'occasion (Montréal, Société des Éditions Pascal, 1945) pour la représentation littéraire par excellence du père imprévoyant en milieu ouvrier, dans ce cas dans les années 1940. Voir aussi les travaux de Martin Petitclerc sur les sociétés de secours mutuels du XIX siècle, notamment là où il fait ressortir la progression d'une nouvelle logique assurantielle dans la période après 1870 , celle de la montée de l'industrie canadienne et québécoise de l'assurance-vie commercialisée. Martin Petitclerc, "Nous protégeons l'infortune». Les origines populaires de l'économie sociale au Québec (Montréal, VLB éditeur, 2007), chapitre 8, 185-215 et «De la providence à la prévoyance : les classes populaires et la société libérale au Québec», dans Jean-Marie Fecteau et Janice Harvey (dir.), La régulation sociale entre l'acteur et l'institution. Pour une problématique de l'interaction (Montréal, Presses de l'Université du Québec, 2005), 236-253. 
Figure 3

Le père prévoyant

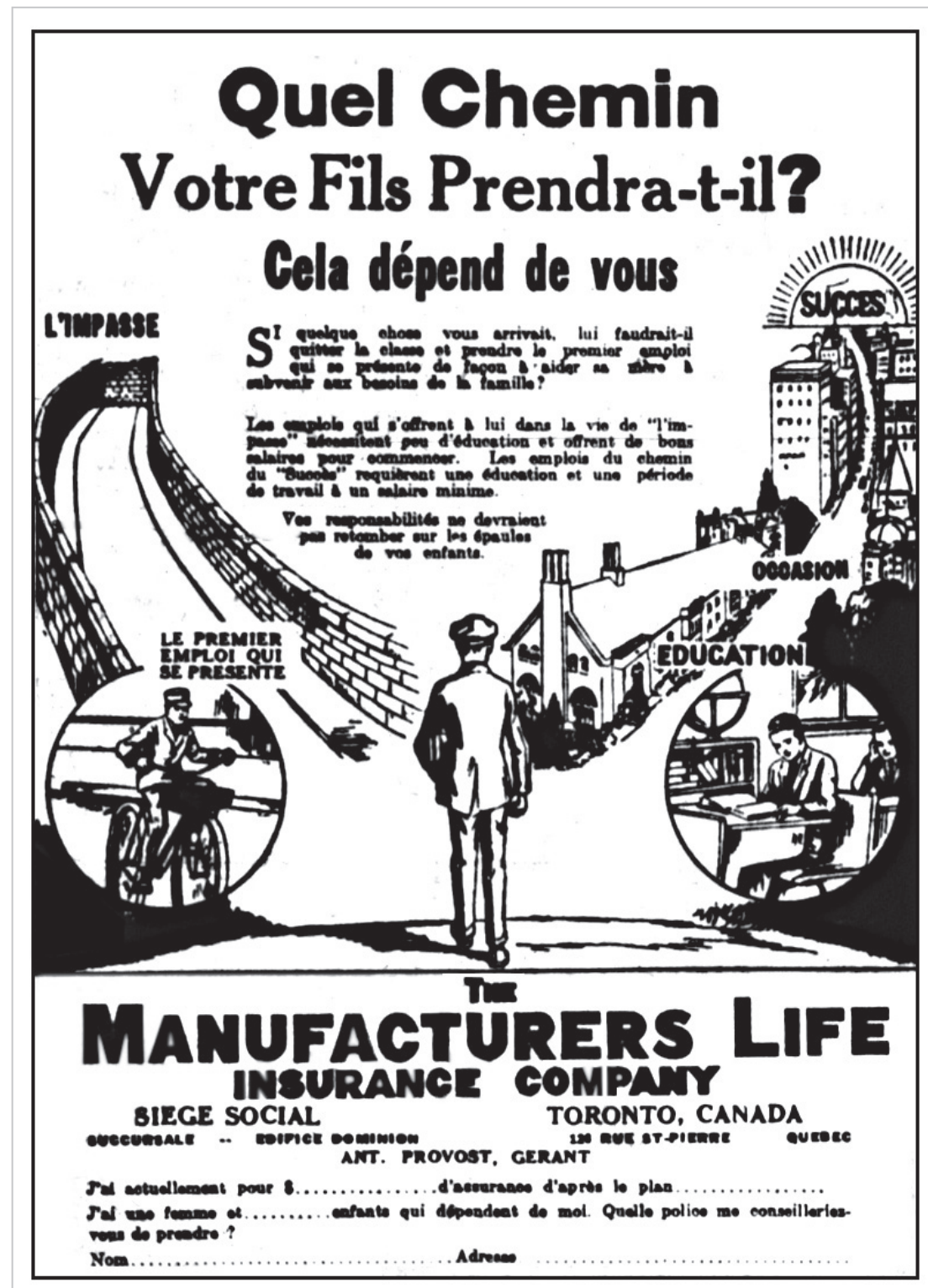

Source: Annonce publicitaire de la Manufacturers Life Insurance Company, Le Soleil, le 14 juin 1924, 7 . 
Figures 4 et 5

\section{Le père prévoyant}

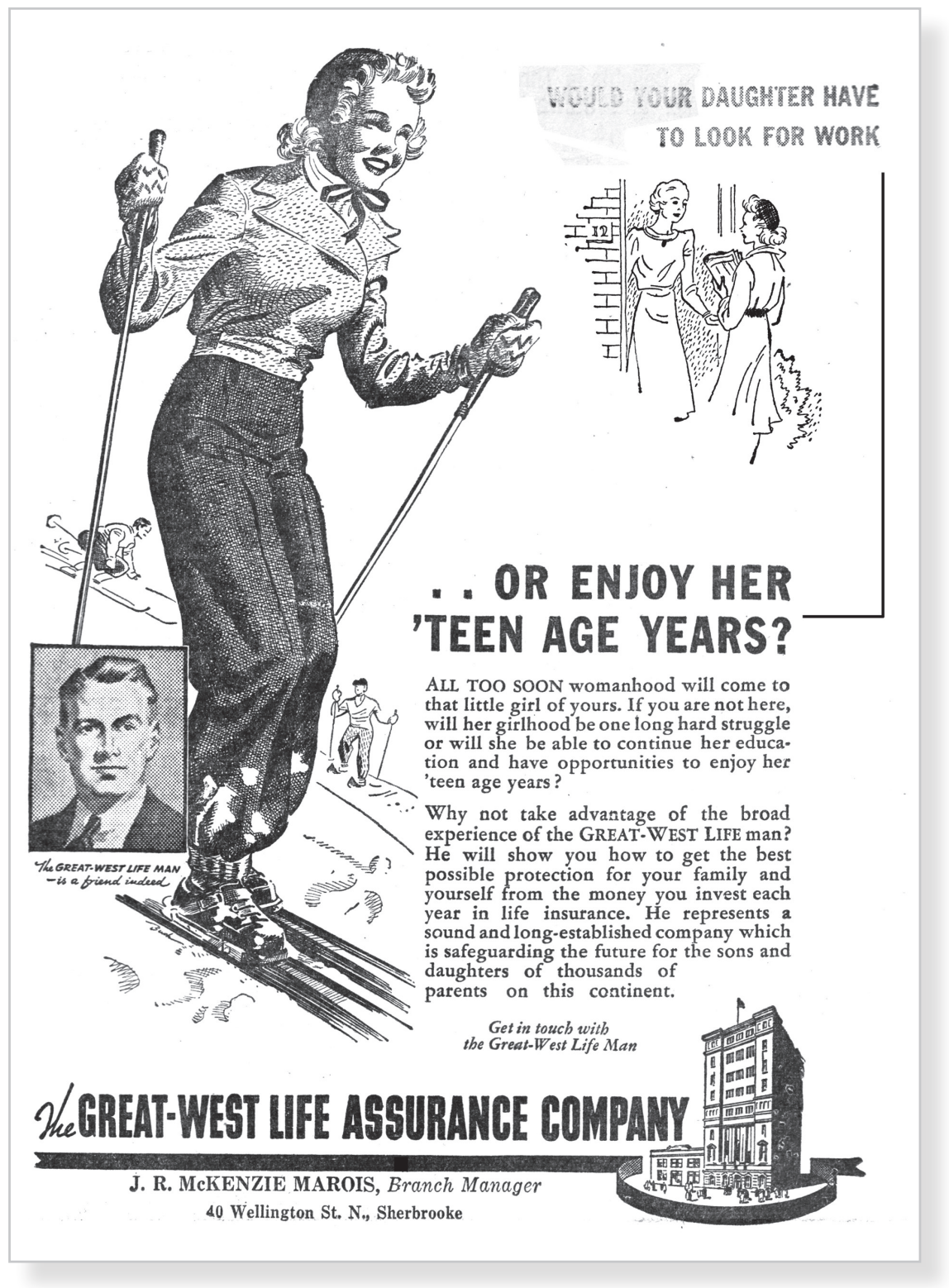

Source: Annonce publicitaire de la Great-West Life Assurance Company, Sherbrooke Daily Record, le 5 décembre 1939, 9. 


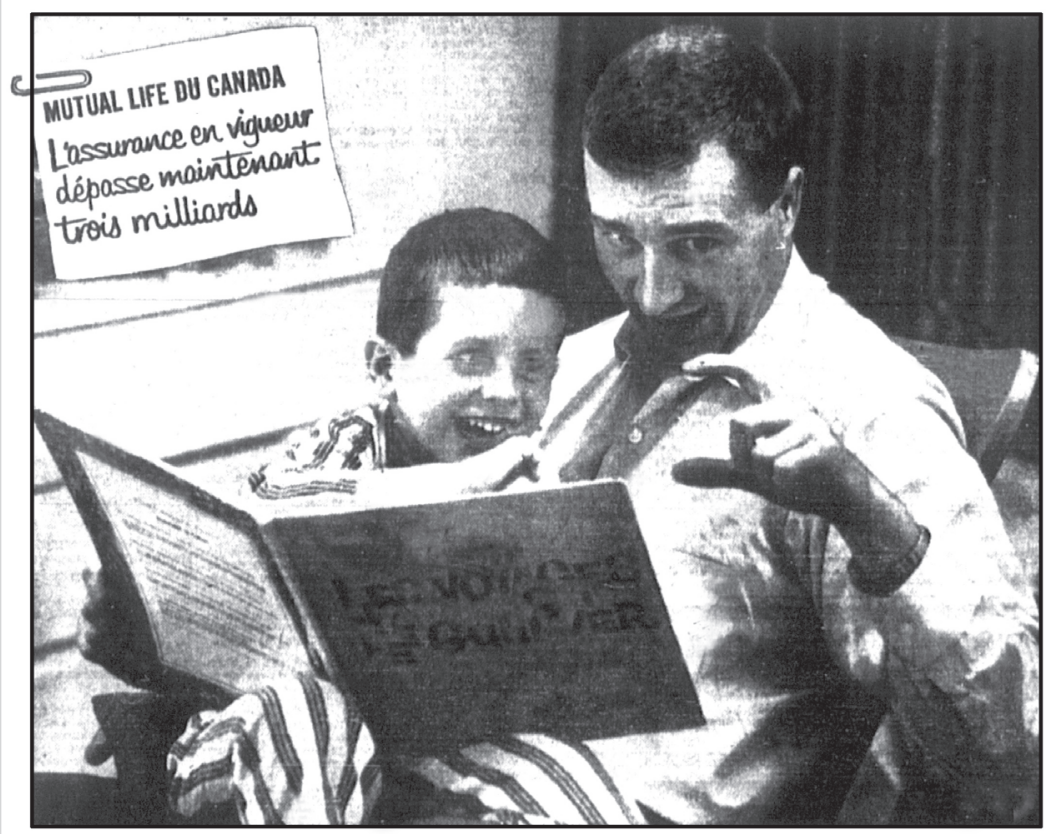

Les papas qui savent toute l'histoire choisissent l'assurance-vie qui rapporte le plus

Aimeriez-vous poséder des centaines de dollars de plus lorsque, le besoin de protéger votre famille étant devenu moins urgent, .votre assurance-vie représentera de l'argent liquide ou une rente pour voa vieux jours? Votre police Mutual Life du Canada peut vous rapporter de plus beaux revenus bien au-delà de ce que vous aurez versé en primes!

Ce revenu supérieur provient des foris dividendes de la Mutual Life. D'année en année, ha Mutual Life du Canada accorde des dividendes exceptionnellement élevés, Lorsquion

les laisae s'accumuter a intérêt, cen dividendes annueis déjà remarquables rapportent de beaux revenus. La valeur a la retraite d'une police de Mutual Life avec dividendes représente aujourd' hui, pour vous, I'un des placements les plus profitables au Canada.

Informez-vous done comment vous, personnellement, pouvez en benéficier. L'agent de la Mutual Life du Canada vous renseignera volontiers, ou écrivez au siège nocial a Waterloo, Ont.
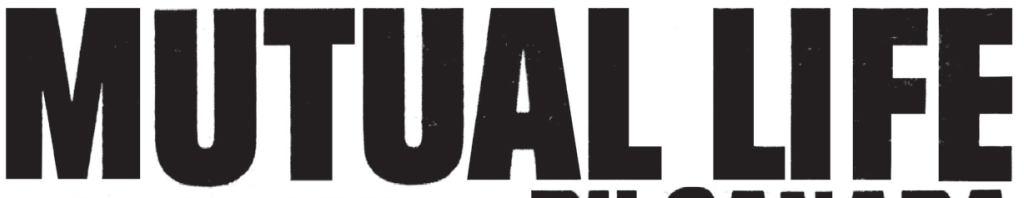

En tete, par un long passe de dividendes exceptionnels

Deux succursales à Québec : 350 est boul. Charest

\section{A. Damars, C.L.U., gérant} VA.) LA 2.4047

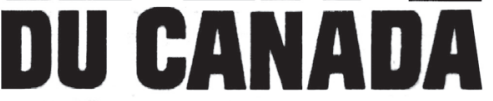

Paul Rony, C.L.U., gérant Ta.) \& 2.4047

Source: Annonce publicitaire de la compagnie d'assurance Mutual Life du Canada, Le Soleil, le 25 juin 1960, 19. 
contredit la raison d'être des polices d'assurance-vie qui sont de plus en plus répandues durant cette période, instruments dont l'objectif est d'assurer au détenteur qu'un pourvoyeur substitut (à savoir la compagnie d'assurances) soit mis en place dans l'éventualité de sa mort ${ }^{46}$. C'est donc lui, ce père qui se veut prévoyant, qui est ciblé dans toute une gamme d'annonces publicitaires publiées en grand nombre dans les journaux quotidiens, annonces qui regorgent de discours et de prescriptions en ce qui concerne la prudence, la planification, bref la prévoyance d'un bon père de famille, pour reprendre cette formulation juridique si bien répandue à l'époque.

Un premier exemple (figure 3) est publié dans le quotidien Le Soleil en juin 1924. Le texte, adressé directement au père de famille, invite celui-ci à réfléchir et à se préparer en fonction de son propre décès. Qu’adviendrait-il de son fils - avec ou sans l'achat d'une police d'assurance-vie de la Manufacturer's Life - si un tel malheur devait se produire?

Si quelque chose vous arrivait, lui faudrait-il quitter la classe et prendre le premier emploi qui se présente de façon à aider sa mère à subvenir aux besoins de la famille? Les emplois qui s'offrent à lui dans la vie de «l'impasse» nécessitent peu d'éducation et offrent de bons salaires pour commencer. Les emplois du chemin du "Succès» requièrent une éducation et une période de travail à un salaire minime. Vos responsabilités ne devraient pas retomber sur les épaules de vos enfants ${ }^{47}$.

Des arguments semblables figurent dans la plupart des annonces que nous avons repérées. La North American Life, par exemple, attire le lecteur du Soleil en 1939 avec une image touchante d'un père marchant main dans la main avec son jeune fils de deux ou trois ans. Sous le titre «Mon Papa» se trouve le texte suivant:

La main confiante d'un petit enfant symbolise la responsabilité qui repose sur les épaules de tout père de famille - la protection et le bien-être qu'il doit à ceux qui lui sont chers. Quel est le papa qui ne consentirait pas de mettre de côté $25 \$$ par jour pour assurer à sa famille, en cas de mort prématurée, un revenu de $50 \$$ par mois durant 10 ans $^{48}$ ?

46. L'histoire de l'assurance-vie au Québec reste largement en friche mais pour deux études intéressantes, voir Claude Cardinal, De la fraternité au conglomérat. Une histoire des compagnies d'assurance-vie québécoises, 1850-1995 (Montréal, Guérin, 2010) et Peter Baskerville, "The Worth of Children and Women: Life Insurance in Early Twentieth-Century Canada », dans Gordon Darroch (dir.), The Dawn of Canada's Century: Hidden Histories (Montréal et Kingston, McGill-Queen’s University Press, 2014), 452-480.

47. Annonce de la Manufactuer's Life, Le Soleil, 14 juin 1924, 7. L'annonce identique avec texte en anglais est publiée le même jour dans le Sherbrooke Daily Record, 14 juin 1924, 6.

48. Annonce de la North American Life, Le Soleil, 27 juin 1939, 1. 
De tels investissements permettent à un père de s'assurer que ses fils adolescents ou jeunes adultes n'auront pas à prendre sa place de pourvoyeur dans une famille devenue monoparentale à la suite de son décès. La même logique peut s'appliquer dans le cas des filles, même si ce cas paraît plus rarement dans nos sources. Citons tout de même une annonce publiée dans le Sherbrooke Daily Record en 1939 (figure 4). "Would your daughter have to look for work», cher Papa, dans le cas de votre décès prématuré? Ou bien, serait-elle en mesure de profiter pleinement de sa jeunesse, en faisant du ski alpin, par exemple, ou d'autres activités de plein air? "All too soon womanhood will come to that little girl of yours. If you are not here, will her girlhood be one long, hard struggle or will she be able to continue to enjoy her "teen age years" 49 "? Il vaut mieux ne pas prendre le risque: achetez donc une assurance-vie chez Great Western Life Assurance Company. Et si vous êtes chanceux, vous vivrez jusqu'à un âge bien avancé et votre assurance pourra être convertie en "argent liquide ou une rente pour vos vieux jours" vous apportant de «beaux revenus - bien au-delà de ce que vous aurez versé en primes " ${ }^{50}$.

Ces quelques exemples, présentés ici en rafale, nous permettent d'identifier le visage d'un père prévoyant tout à fait soucieux de l'avenir de ses enfants, qui auront droit à ses ressources et à sa protection même après sa mort, et dont l'amour paternel se traduit par l'achat d'une police d'assurance-vie. Les images utilisées pour véhiculer cette logique sont, par ailleurs, tout aussi parlantes que les textes publicitaires. On voit des pères généreux et impliqués, faisant des gestes de tendresse, offrant des encouragements dans le jeu et aux loisirs ou lisant à haute voix un livre préféré à l'heure du coucher (figure 5). C'est une autre facette du modèle du père éducateur que nous avons évoqué ci-haut, croisé ici avec un certain visage de père consommateur dont les images publicitaires - pour les assurances et d'autres services financiers, certes, mais aussi pour les voyages, les automobiles et les articles de sport - sont parmi les sources les plus éloquentes et les plus largement consultées par les chercheurs dans ce domaine ${ }^{51}$.

49. Annonce de la Great Western Life Assurance Company, Sherbrooke Daily Record, 5 décembre 1939, 9.

50. Annonce de la Mutual Life du Canada, Le Soleil, 25 juin 1960, 19 (figure 5).

51. Voir notamment M. Roy, L'évolution de la figure paternelle..., op. cit., et R. Rutherdale, «Fatherhood and Masculine Domesticity During the Baby Boom», loc. cit.. Au total, plus de 10 p. cent des annonces publicitaires dans notre corpus (32 sur 286) portent sur les polices d'assurance-vie, placées pour la plupart par les grandes compagnies d'assurances canadiennes et américaines. Aucune de ces annonces n'est publiée avant 1921, mais elles prennent ensuite une place assez constante. Dès lors, on compte toujours une forte proportion d'annonces sur les polices d'assurances-vie parmi les publicités où figure un père de famille. Parmi les autres catégories importantes d'annonces, mentionnons celles portant sur les aliments (céréales, chocolat, friandises), les boissons (bière, whisky, liqueurs douces), les vêtements, certains 
Figure 6

\section{Le père sportif}

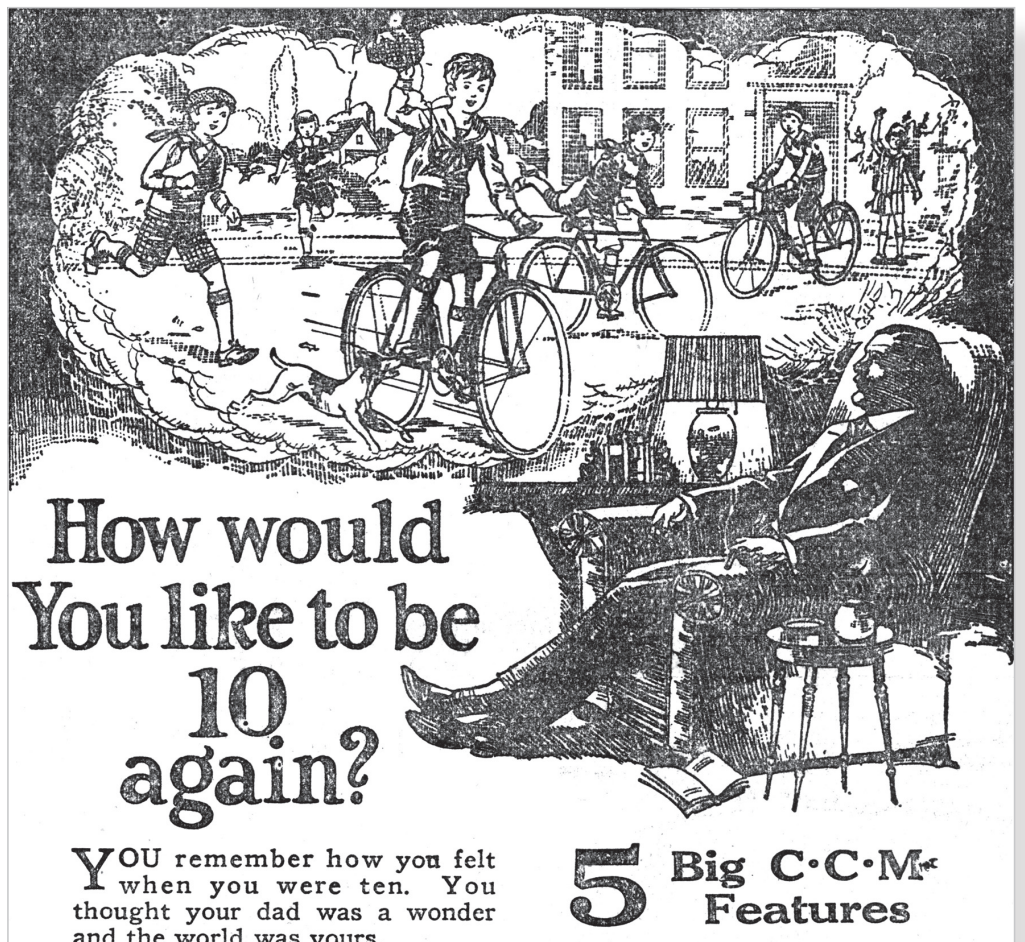
and the world was yours.

Your boy wants a bicycle-A C.C.M. Most of the boys have them or are getting them, the boys with dads like your boy's dad. Ask him, and see if this is not so.

If you let him choose he'll choose a C.C.M.

The C.C.M. is a quality bicycle. It's the machine he wants-the bicycle with the Triplex Hanger. You know the satisfaction of a new bicycle when the hanger or crankshaft runs true and.smoothly. The Triplex Hanger stays that way.

Ask the C.C.M. dealer to tell you about the easy payment plan.

The C.C.M. is a quality bicycle. It is the wheel with

1 The Triplex Hanger, for smooth, easier pedalling.

2 The Hercules Coaster Brake, the "little fellow with the giant grip".

3 Gibson Pedals, one piece, aluminum frame, rustproof.

4 Dunlop Tires, fully guaranteed.

5 Twenty-year nickel-plating.

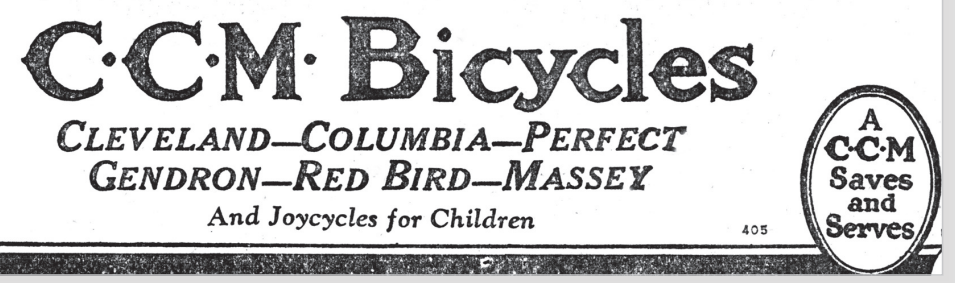

Source: Annonce publicitaire de la compagnie de bicyclettes C.C.M., Sherbrooke Daily Record, le 13 juin 1927, 8. 


\section{Le père sportif}

Le visage du père sportif, enfin, nous amène sur un autre terrain complètement, ou peut-être sur plusieurs terrains à la fois, car le visage du père sportif se décline au pluriel, tellement ses formes sont multiples. L'une des grandes catégories d'annonces publicitaires qui ciblent les pères de famille (nous venons de le souligner) est celle des articles de sport, conçus ici dans le sens large des activités physiques en plein air. Nous nous limiterons au seul exemple reproduit à la figure 6 : une annonce pour les vélos C.C.M. qui date de 1927 et qui invite un père bourgeois, de 35 ou 40 ans, assis confortablement dans son fauteuil, à songer à sa propre jeunesse et au sentiment d'avoir 10 ans.

You remember how you felt when you were ten. You thought your dad was a wonder and the world was yours. Your boy wants a bicycle - A C.C.M. Most of the boys have them or are getting them, the boys with dads like your dad. Ask him, and see if this is not so [...]. You know the satisfaction of a new bicycle when the hanger or crankshaft runs true, and smoothly ${ }^{52}$.

Alors, pour susciter chez son propre garçon ce sentiment de joie dans le mouvement d'une machine de qualité et, encore davantage, ce sens de l'émerveillement face à un monde de possibilités, ouvertes notamment par un père généreux et bienveillant, quoi de mieux que de lui acheter un vélo C.C.M.? La bicyclette, par ailleurs, figure parmi les symboles les plus évocateurs de l'enfance masculine urbaine au $\mathrm{XX}^{\mathrm{e}}$ siècle, fournissant aux garçons non seulement une mesure de mobilité dans la ville, mais aussi un apprentissage dans la performance de certains attributs (autonomie, force, vitesse, maîtrise de la technologie) associés à la masculinité à l'époque $^{53}$. Le fait que ces qualités, au moins en principe, soient transmises de père en fils n'est pas sans importance pour notre analyse.

Si la bicyclette est associée surtout aux loisirs urbains à cette époque, le goût chez les citadins de passer du temps à la campagne, profitant de la nature et du plein air, est l'une des grandes tendances du $\mathrm{XX}^{\mathrm{e}}$ siècle, associée au mouvement "antimoderniste» bourgeois et documentée

appareils électriques (radio, phonographe, téléphone), les articles de sport, les voyages et les automobiles. À noter que les périodiques consultés dans le cadre de cette recherche sont deux quotidiens (Le Soleil et le Sherbrooke Daily Record) et trois revues populaires: Le Samedi, La Revue moderne et La Revue populaire.

52. Annonce publicitaire de la compagnie C.C.M., Sherbrooke Daily Record, 13 juin 1927, 8.

53. Dans une étude sur Windsor, Ontario, Christopher Greig souligne l’importance du vélo comme symbole de pouvoir et de liberté pour la jeunesse masculine. C. Greig, «Boys and Boyhood: Exploring the Lives of Boys in Windsor, Ontario During the Postwar Era, 1945-1965 ", dans Gossage et Rutherdale (dir.), Masculine Histories in the Making, op. cit., à paraître. 
abondamment dans les études récentes sur la villégiature ${ }^{54}$. Une autre variation du père sportif serait donc le père plein air et l'un des beaux exemples serait Victor Morin, dont l'une des joies profondes était liée à sa maison d'été située "sur le versant du Mont-Bruno" au tout début du $\mathrm{XX}^{\mathrm{e}}$ siècle. Selon le témoignage de sa fille Renée,

[I]1 savait laisser derrière la porte verrouillée de son bureau, les soucis de l'homme d'affaires, et jouir de la campagne en toute sérénité. Une promenade à cheval ou à pied en compagnie des enfants; des excursions de pêche ou une escalade dans la montagne le dimanche; un peu de camping à Chambly ou ailleurs au bord du Richelieu avec les fils aînés, tout cela constituait pour lui des loisirs très réconfortants ${ }^{55}$.

La pêche, le camping et la navigation de plaisance sont par ailleurs des thématiques qui reviennent régulièrement dans notre corpus d'annonces publicitaires, notamment celles publiées en juin pour souligner la fête des Pères et préparer les vacances estivales.

Une autre variation du père sportif serait le père amateur ou fan, celui qui a le goût, les moyens et le temps d'assister aux nouveaux divertissements commercialisés de l'époque, dont les sports-spectacles comme le hockey professionnel ou semi-professionnel. Le fait d'emmener son fils, ou plus rarement sa fille, à de tels événements pouvait inspirer de fortes émotions et à long terme de riches souvenirs chez le jeune en question. Paul L’Anglais (1907-1982), qui passe son enfance à Québec dans un milieu bourgeois, se rappelle de l'énorme plaisir qu'il a ressenti lorsqu'il assistait aux matchs du club local, les Bulldogs, avec son père:

Aller voir une partie de hockey, c'était une traite extraordinaire et je me souviens, quand j'avais cinq ou six ans, que papa m'y a emmené deux ou trois fois. Il fallait s'habiller chaudement et apporter avec soi un réchaud qu'on appelait chinois [...] La glace était naturelle et il fallait être amateur de hockey, comme l'était papa et comme je le suis devenu, pour aller endurer ce froid-là pour le plaisir de voir un match. Il n’y avait pas évidemment de radio, ni de télévision pour nous faire entendre ou voir confortablement ce divertissement ${ }^{56}$.

Ces réflexions et souvenirs personnels pourraient sans doute être multipliés. Les circonstances et les détails, fort variables, comptent moins que la trame

54. Pour le Québec, voir notamment Michèle Dagenais, «Fuir la ville: Villégiature et villégiateurs dans la région de Montréal, 1890-1940", Revue d'histoire de l'Amérique française 58, 3 (janvier 2005): 315-345.

55. R. Morin, Un bourgeois d'une époque révolue, op. cit., 99.

56. Paul L’Anglais, Ma belle époque (Québec, Les Éditions Héritage, 1992), 67. Pour un témoignage féminin assez remarquable dans la même veine, voir S. Monet-Chartrand, Ma vie comme rivière, op. cit., 117. 
centrale du récit, celle où un père partage avec son enfant des divertissements excitants, que ce soit un match de hockey ou un autre type de sortie sportive ou de spectacle. Le père participe ainsi à l'initiation de l'enfant ou de l'adolescent au monde extérieur, en dehors du foyer et de l'influence maternelle, et donc à la cité et à son rôle éventuel de citoyen ou de citoyenne ${ }^{57}$. Cela est tout à fait cohérent avec l'émergence, soulignée par certains chercheurs, du père copain: celui qui s'implique plus activement qu'autrefois auprès de ses enfants, mais dont le rôle reste essentiellement récréatif ou ludique et circonscrit aux temps libres de cet homme qui conserve à l'époque, rappelons-le, son rôle de pourvoyeur ${ }^{58}$.

\section{CONCLUSION}

L'expression "agir en bon père de famille» est bien connue au Québec. Elle réfère certes à un comportement exprimé à l'intérieur de la sphère familiale mais, plus largement, elle renvoie à une façon d'être en société qui s'avère conforme aux attentes spécifiques d'une culture à l'endroit des hommes. À notre sens, agir en bon père de famille c'est agir avec soin, responsabilité, prudence et jugement: c'est donc répondre essentiellement aux mêmes défis que ceux évoqués par Rutherdale avec son concept central de responsible family manhood. Les idées fortes que sont la responsabilité et le souci de sa famille, le tout formant une dimension d'une identité masculine particulière, restent relativement stables, nous pourrions le croire, durant la période qui nous intéresse, même si le père éducateur (dans la logique des trois modèles évoqués ci-haut) fait du chemin par rapport au père pourvoyeur. Mais les manières de répondre à ces attentes et aux prescriptions qui les appuient sont nombreuses et variables d'un homme à l'autre, en fonction de toute une gamme de facteurs, dont l'âge, la génération, le milieu (rural ou urbain), la classe sociale et la communauté d'appartenance.

Il existe donc un éventail de réponses potentielles au défi et à la demande sociétale d'agir «en bon père de famille». Nous l'avons souligné avec la métaphore des visages du père empruntée à Rutherdale et à Demos. Comment les biographies des pères québécois «interagissent-elles avec les temps et les

57. Merci à Louise Bienvenue et aux membres de son séminaire en histoire des identités à l'Université de Sherbrooke de m'avoir proposé cette piste d'analyse en février 2016.

58. Laura King résume ainsi son analyse de ces enjeux dans le contexte britannique de la même époque: «From a child's perspective, fathers thus represented the more "fun" side of parenting; for most men, whilst at home they were at leisure, whereas the home represented a place of work for women. That fathers were the parent who played and entertained underlines the fact that childcare as a chore remained the responsibility of mothers; parenting was part of men's leisure time rather than labour in itself », L. King, Family Men..., op. cit., 55. 
lieux où ils prennent racine, où ils grandissent, arrivent à l'âge adulte, et deviennent père ${ }^{59}$ " ? Voilà un questionnement riche et prometteur qui pourra ouvrir de nouvelles pistes en histoire des pères québécois. Il nous permet, dans un premier temps, de mettre l'accent (davantage, au moins) sur les expériences paternelles, dans un contexte où les normes, les attentes, les conseils et les prescriptions semblent parfois prendre le haut du pavé. Il nous permet, dans un deuxième temps, d'éviter deux dangers réels : celui souligné en 2006 par Vacante de se lancer dans une opération de récupération qui négligerait les rapports de force qui sous-tendent le pouvoir masculin dans cette société patriarcale ${ }^{60}$; et celui que nous avons souligné plus haut, du recours exclusif à des schémas conceptuels globalisants, trop lourds et trop sommaires pour tenir compte d'une réalité complexe et variable où les interactions entre les spécificités québécoises et les généralités nord-américaines (voire occidentales) doivent être prises en compte.

Le recours à la grille des visages de la paternité nous permet, par ailleurs, d'ajouter de nouvelles dimensions à nos analyses de la paternité québécoise avant 1960, période associée à la diffusion graduelle d'une façon plus moderne et plus libérale d'être père au Québec, mais sans que les éléments fondamentaux de la famille patriarcale - dont la division sexuelle des tâches et la conception hiérarchisée du genre - ne soient réellement contestés. Il existe au moins un visage de la paternité - érigé souvent, on l'a vu, en "modèle» - qui reste essentiellement stable dans la période traitée ici (1900-1960), à savoir celui du père pourvoyeur. Disons-le plus directement: l'obligation de faire vivre ses enfants en leur fournissant, normalement par son travail quotidien, des aliments, des vêtements, un abri, une éducation et certains conforts de base font partie des responsabilités masculines et paternelles les plus fondamentales tout au long de la période étudiée. Dans cette optique, il faut comprendre l'émergence de nouveaux visages de paternité comme de nouvelles composantes de la parentalité masculine: des couches qui s'ajoutent mais sans jamais remplacer ou éliminer cette responsabilité paternelle de base.

Or, avec l'appui des récits de vie et des annonces publicitaires cités ci-haut, nous sommes en mesure d'affirmer que la «modernisation» de la paternité au Québec, qui est en cours sans contredit avant 1960, comporte entre autres le déclin, voire la disparition graduelle de certains visages de la paternité comme celui du père spirituel, qui offre annuellement sa bénédiction au nom de Dieu, ou bien celui du père disciplinaire, qui croit fermement au dicton "qui aime bien châtie bien ». Cette modernisation préside aussi à l'émergence ou à la généralisation d'autres visages, comme ceux du père prévoyant et du

59. Traduction de R. Rutherdale, "Three Faces of Fatherhood as a Masculine Category ", loc. cit., 325.

60. J. Vacante, «Liberal Nationalism and the Challenge of Masculinity Studies», loc. cit., 106. 
père sportif que nous avons explorés en détail ci-haut, ou d'autres que nous avons tout simplement mentionnés au passage, comme ceux du père consommateur et du père copain. Tout cela se situe par ailleurs dans un contexte spécifiquement québécois où l'influence de l'Église catholique reste forte, où le Code civil persiste dans sa définition traditionnelle de la puissance paternelle, où la baisse de la fécondité maritale se déroule très graduellement avant 1960 (comment donc être le père "moderne» d'une famille de taille et de structure "traditionnelle»?) et où l'accès aux revenus et aux temps libres, pour les hommes de la classe ouvrière d'origine canadienne-française, irlandaise, italienne ou autre, est très loin d'être acquis et compromet l'épanouissement des visages de père éducateur, de père consommateur, de père sportif ou de père copain malgré l'attrait qu'ils peuvent représenter.

Nous terminons donc en réitérant notre argument principal selon lequel la grille des "visages de la paternité» nous permet de dépasser le schéma des «modèles» qui peuvent, certes, être en évolution dans la longue durée mais dont la lourdeur fait en sorte que les mouvements plus subtils risquent de passer inaperçus. Mieux vaut une analyse dynamique, souple et multidimensionnelle que permet ce concept des visages, donc des comportements potentiels pour les «responsible family men»: ces hommes qui partagent, grosso modo, une vision commune de l'idéal du bon père de famille, sans toujours pouvoir l'atteindre. Avec les exemples du père spirituel, du père disciplinaire, du père prévoyant et du père sportif-tels que nous les avons identifiés dans nos sources - nous croyons fournir une esquisse de ce à quoi une telle analyse pourrait ressembler pour la période 1900-1960.

Il ne s'agit là, bien sûr, que de quelques exemples. Bien d'autres pourraient s'ajouter à la liste, qu'il s'agisse de visages positifs - il y a de nombreux père héros dans notre corpus, par exemple, ainsi que certains grands-pères particulièrement bienveillants - ou négatifs: pères absents, pères violents, pères alcooliques, pères paresseux ou, au contraire, pères bourreaux de travail, et peut-être bien d'autres, à juger notamment par les études littéraires, là où la figure paternelle brille surtout par sa faiblesse ${ }^{61}$. Pour la période plus récente, disons depuis les années 1970, les transformations dans ce domaine sont plus rapides et plus fondamentales que durant les six décennies qui nous préoccupent. Comment s'étonner, à ce moment, si de nouveaux visages du père québécois sont si nombreux et si variés de nos jours, à l'époque dite du "nouveau père "? Nous pensons notamment ici au père au foyer qui s'occupe de ses enfants à temps plein, soit pendant quelques mois durant un congé parental, soit à plus long terme, ou bien au père militant qui manifeste publiquement sa colère contre 
les décisions judiciaires qui réduisent ou éliminent son accès aux enfants d'une union rompue, érigées en tendance lourde par des organismes à forte tendance antiféministe comme Fathers4Justice. Ce sont là des pistes de réflexion qui pourraient, nous l'espérons du moins, inspirer des recherches sur les visages de la paternité dans la période contemporaine. Elles invitent aussi à une plus fine exploration du XIX ${ }^{\mathrm{e}}$ siècle, période largement négligée par les chercheurs du domaine, en permettant la prise en compte de la diversité et du dynamisme des expériences paternelles sans les réduire au modèle sommaire et approximatif du père pourvoyeur. 